\title{
Non-Phase Matched Stimulated Parametric Scattering in Semiconductor Plasma: Temporal Effects
}

\author{
S. Ghosh, Swati Dubey and Kamal Jain* \\ School of Studies in Physics, Vikram University, Ujjain, Madhya Pradesh, 456001, India \\ (Received February 12, 2018; in final form February 27, 2019)
}

\begin{abstract}
Present paper is an effort to study stimulated parametric scattering of an electromagnetic wave propagating in a doped semiconductor plasma medium. We study theoretically the time evolution of a parametrically amplified wave in a non-phase matched semiconductor plasma medium. In order to describe the dynamics of the system, Maxwell's equations and hydrodynamic model for one component plasma along with coupled mode theory are used. These equations are solved numerically in the whole time range up to the steady state for a crystal. Following standard approach expressions for second order optical susceptibility, steady state, and transient gain coefficient for stimulated parametric scattering have been derived. Numerical estimation is made by considering an $n$-CdS irradiated by an intense laser at $77 \mathrm{~K}$. This study suggests design of an optical parametric amplifier with tunable temporal characteristics, which are found to agree well with the experimental findings.
\end{abstract}

DOI: 10.12693/APhysPolA.135.363

PACS/topics: 42.65.-k, 42.65.An, 52.38.-r, 52.38.Dx

\section{Introduction}

Optical properties of semiconductors are greatly modified, when electrons are excited optically. This fact makes semiconductors suitable for optical memories and switching elements. Moreover, their properties could be tailored easily by fields, compositions, and micro-structuring; therefore they are much often used for the manipulation of light through nonlinear interaction. Nonlinear interactions are generally used either to shift the available optical frequency, or to remodel spatio-temporal characteristics of coherent light beam. Change in the spatiotemporal profile and polarization state without touching its carrier frequency demands absence of phase matching condition practically. In order to select dominant nonlinear interaction in given experimental situations, it is essential to maintain phase matching conditions. Material dispersion results in momentum mismatch in many energy conserving nonlinear optical interactions [1-3].

However, one way to deal with the momentum mismatch situation is to use birefringent phase matching, angle phase matching, and anisotropic techniques [4-6]. Spatio-temporal nonlinear diffraction for quasi-phase matching of momentum and energy mismatch has been introduced for beam manipulation and multi-harmonic generation. Concepts of dispersion engineering and dispersion compensation has been utilized for phase mismatch switching with a periodic profile $[7,8]$. Nowadays metamaterials with zero refractive index are used to eliminate the requirement of phase matching which allows improved efficiency of nonlinear process regardless of directionality [9]. Effect of phase mismatch has also been investigated in negative index material [10],

*corresponding author; e-mail: jainrupam1987@gmail.com although fabrication of desired bulk metamaterials involves certain limitations due to complexity in design. In optically isotropic materials, having strong phononphoton coupling, low frequency index is quite high, and phase matching is not possible in the forward direction [11]. Even quasi phase matching techniques are not applicable to such cubic crystals.

In view of above limitations and keeping in mind the fact that phase mismatch is more relevant to feasible experimental conditions, it seems to be more realistic to consider a tolerable small phase mismatch in the phenomena of interest. Therefore, apart from using metamaterials and all other phase matching solutions, present study for the first time focuses on an analytical investigation of non-phase matched stimulated parametric scattering (SPS) in a cubic compound semiconductor plasma medium. Relevant data of an ionic crystal $(\mathrm{CdS})$ is used for the numerical appreciation of the analysis. The crystal sample is assumed to be shined by a laser with photon energy well below the band gap of the crystal, so that only free charge carriers influence the optical properties of interest.

\section{Theoretical formulations}

This section considers SPS under the influence of an intense laser beam travelling along $x$ axis in a nonlinear crystal. The electric field associated with pump wave under plane wave approximation may be expressed as $\boldsymbol{E}_{\mathbf{0}}=E_{0}(\hat{x}) \exp \left(\mathrm{i}\left(k_{0} x-\omega_{0} t\right)\right)$. SPS, a three-wave mixing process gets initiated by a single intense pump field at frequency $\omega_{0}$ at the input to a nonlinear crystal. This field is provided by the laser source which in turn mixes through the nonlinear susceptibility with a signal field at $\omega_{1}$ to give rise to an idler field generated as a result of nonlinearity of the medium. The idler field so generated mixes back with the pump to produce additional signal which remixes with the pump to produce more idler. The process continues in this way until power is gradually 
transferred from the strong pump to the initially weak signal and idler fields through the nonlinear interaction mediated by second order susceptibility $\chi^{(2)}$. It is customary to describe this interaction macroscopically by means of the nonlinear polarisability, which is nonzero in piezoelectric crystal [12]. Hence, in the presence of the strong input pump field, signal and idler fields can experience growth as they propagate through the nonlinear crystal.

Hydrodynamic model for $n$-type homogeneous semiconductor plasma medium of infinite extent at liquid nitrogen temperature has been considered, which restricts validity of our analysis only in the limit $k_{a} l \ll 1$ ( $l$ is the carrier mean free path). The coupled mode scheme is applied to obtain a simplified expression for the second order susceptibility via nonlinear polarization. While considering coupled wave problems, the classical description is preferred over quantum one, since the decay or amplification of the waves depends on the relative phases among them, which is very well tackled classically. On the other hand, if the number of quanta is taken into account, the phases well be undetermined as required by the uncertainty principle. Therefore in this paper, a classical discussion on the coupling of acoustic wave and light wave is considered. Generation and amplification of signal and idler are also hoped to be strongly dependent functions of the phase mismatch parameter $\Delta k=\boldsymbol{k}_{\mathbf{0}}-\boldsymbol{k}_{\mathbf{1}}-\boldsymbol{k}_{\boldsymbol{a}}$, where $\boldsymbol{k}_{\mathbf{0}}, \boldsymbol{k}_{\mathbf{1}}$, and $\boldsymbol{k}_{\boldsymbol{a}}$ are waves vectors of pump, signal, and acoustic modes, respectively. Inclusion of phase mismatch factor addresses the major problem that occurs due to inherent phase mismatch between the interacting waves propagating through the nonlinear materials due to material dispersion. The frequency of scattered light is assumed to vary as $k_{a}=k_{0} \sqrt{2(1+\cos \theta)}$, where $\theta$ is scattering angle. However, according to scattering theory, amplification of only spectral component shifted towards shorter or longer wavelengths, relative to the spontaneous three photons, is possible [13].

Following the standard approach $[14,15]$, here after referred as paper I and II and basic equations for the necessary formulations,

$$
\begin{aligned}
& \frac{\partial \boldsymbol{\vartheta}_{0}}{\partial t}+\left(\nabla \cdot \boldsymbol{\vartheta}_{0}\right) \boldsymbol{\vartheta}_{0}+v \boldsymbol{\vartheta}_{0}=\frac{e}{m} \boldsymbol{E}_{0}, \\
& \frac{\partial \boldsymbol{\vartheta}_{1}}{\partial t}+\left(\nabla \cdot \boldsymbol{\vartheta}_{1}\right) \boldsymbol{\vartheta}_{0}+\left(\nabla \cdot \boldsymbol{\vartheta}_{0}\right) \boldsymbol{\vartheta}_{1}+v \boldsymbol{\vartheta}_{1}=\frac{e}{m} \boldsymbol{E}_{1} .
\end{aligned}
$$

Response of the charge carrier to the incident pump field is given by zero-order momentum transfer Eq. (1) in which $\boldsymbol{\vartheta}_{0}$ is the zeroth-order velocity of the electrons. Similarly Eq. (2) represents the first order equation of motion of electrons with first-order fluid velocity $\boldsymbol{\vartheta}_{1}$. The components of the oscillatory electron fluid velocity in the presence of pump fields are obtained from Eq. (1) along $x$ and $y$ direction, respectively

$$
\vartheta_{0 x}=\frac{\bar{E}}{\left(v-\mathrm{i} \omega_{0}\right)}, \quad \vartheta_{0 y}=\frac{\bar{E}}{\omega_{0}^{2}}
$$

where $\bar{E}=\frac{e}{m} E_{0}$.
The other equations used in this analysis are

$$
\begin{aligned}
& \frac{\partial n_{1}}{\partial t}+n_{0} \frac{\partial \vartheta_{1}}{\partial x}+n_{1} \frac{\partial \vartheta_{0}}{\partial x}+\vartheta_{0} \frac{\partial n_{1}}{\partial x}=0, \\
& \rho \frac{\partial^{2} u}{\partial t^{2}}+2 \gamma_{s} \rho\left(\frac{\partial u}{\partial t}\right)+\beta \frac{\partial E_{1}}{\partial x}=C \frac{\partial^{2} u}{\partial x^{2}}, \\
& \frac{\partial E_{1}}{\partial x}+\frac{\beta}{\varepsilon} \frac{\partial^{2} u}{\partial x^{2}}=\frac{n_{1} e}{\varepsilon} .
\end{aligned}
$$

Conservation of charge is represented by the continuity Eq. (3) in which $n_{0}$ and $n_{1}$ are the unperturbed and perturbed electron densities. Equation (4) represents motion of lattice vibration in a piezoelectric medium $\rho, u, \gamma_{s}$ and $C$ being mass density of the crystal, displacement of the lattice, phenomenological damping parameter of acoustic mode, and crystal elastic constant, respectively. The space charge field $E_{1}$ is determined from the Poisson Eq. (5) in which the second term on left hand side represents the piezoelectric contribution and $\varepsilon$ and $\beta$ are the scalar dielectric and piezoelectric constant of the semiconductor.

\subsection{Temporal amplification characteristics}

In the parametric scattering process, coupling of the electron plasma wave and generated acoustic wave takes place only through the electronic contribution of nonlinear optical susceptibility of the medium. One may derive expression for the second order optical susceptibility from the equation (8) of paper II and utilize expression for induced nonlinear polarization given by

$$
\begin{aligned}
& P_{1}\left(\omega_{1}\right)=\varepsilon_{0} \chi^{(2)} E_{1}^{*} E_{0}, \\
& \chi^{(2)}=\frac{A e \varepsilon_{1} \varpi_{p}^{2}\left(k_{0}-k_{a}-\Delta k\right)}{2 m \gamma_{s} \omega_{a} \omega_{1} \omega_{0}} \\
& \quad \times\left[\left(\delta_{1}^{2}+\mathrm{i} v \omega_{a}\right)-\frac{\left(2 k_{1}+k_{a}+\Delta k\right)^{2} \bar{E}^{2}}{\left(\delta_{2}^{2}-\mathrm{i} v \omega_{1}\right)}\left(1+\frac{\omega_{1}}{\omega_{0}}\right)\right]^{-1},
\end{aligned}
$$

where $\operatorname{Re} \chi^{(2)}$ and $\operatorname{Im} \chi^{(2)}$ are the real and imaginary parts of susceptibility, respectively. Meaning of all the other symbols are given in paper II.

Our next aim is to investigate the nonlinear absorption coefficient $(\alpha)[16]$ using the following relation:

$$
\alpha=\frac{\left(k_{0}-k_{a}-\Delta k\right)}{2 \varepsilon_{1}} \operatorname{Im}\left(\chi^{2}\right) E_{0},
$$

where

$$
\begin{gathered}
\operatorname{Im} \chi^{(2)}=\frac{A e \varepsilon_{1} \varpi_{p}^{2}\left(k_{0}-k_{a}-\Delta k\right)}{2 m \gamma_{s} \omega_{a} \omega_{1} \omega_{0}} \\
\times \frac{\omega_{1} v\left[\left(2 k_{1}+k_{a}+\Delta k\right)^{2} \bar{E}^{2}-\omega_{1} \omega_{a} v^{2}\right]-\delta_{2}^{4} \omega_{a} v}{\left[\delta_{1}^{2} \delta_{2}^{2}+\omega_{1} \omega_{a} v^{2}-\left(2 k_{1}+k_{a}+\Delta k\right)^{2} \bar{E}^{2}\right]^{2}+\left[\delta_{1}^{2} \omega_{1} v-\delta_{2}^{2} \omega_{a} v\right]^{2}} .
\end{gathered}
$$

The nonlinear steady-state gain of the signal as well as idler waves can be possible only if $\alpha$ obtainable from Eq. (8) is negative. It is evident that the pump amplitude 
should be well above the threshold pump amplitude necessary for the onset of steady state SPS process, which could be derived from Eq. (7) under the condition of $\operatorname{Im} \chi^{(2)}=0$ as

$$
\left|E_{0 t h}\right|=\frac{m}{e\left(2 k_{1}+k_{a}+\Delta k\right)} \sqrt{\frac{\delta_{2}^{4} \omega_{a}+v^{2} \omega_{1}^{2} \omega_{a}}{\omega_{1}}} .
$$

Transient characteristics of nonlinear optical effects influence the rapidity with which various kinds of optical functions can be performed. In view of availability of state of art ultrafast pulsed lasers having pulse duration comparable or smaller than photon life times, it would be interesting to study temporal amplification characteristics of the semiconductor plasma medium. Therefore we will further explore the temporal derivatives, using following expression for transient gain coefficient [17]:

$$
g_{T}=\sqrt{g_{s s} L \Gamma \tau_{p}}-\Gamma \tau_{p},
$$

in which $\Gamma$ is the acoustical phonon life time, $L$ is the interaction length, $\tau_{p}$ is the pulse duration and $g_{s s}$ is the steady state gain coefficient evaluated by Eq. (8). Transient gain coefficient can be determined with the help of Eq. (10) for the SPS phenomenon in a semiconductor plasma. Evolution of SPS in time has been explored considering that the pulse duration of the pump is much shorter than $\Gamma^{-1}$. This transient process will lead to dependence of scattering angles and frequencies on the interaction length and duration of the interaction process.

\section{Results and discussion}

We now address a detailed numerical appreciation of our theoretical model for the study of a non-phase matched SPS through determination of second order optical susceptibility, nonlinear absorption coefficient, steady state gain, and transient gain coefficient in an $n$-type $\mathrm{CdS}$ crystal at $77 \mathrm{~K}$ duly irradiated by a $\mathrm{CO}_{2}$ laser. The physical constants considered for the analysis are: $m=0.107 m_{0}\left(m_{0}\right.$ being the free electron mass), $\varepsilon_{1}=9.35, \gamma_{s}=2 \times 10^{10} \mathrm{~s}^{-1}, \rho=4.82 \times 10^{3} \mathrm{~kg} / \mathrm{m}^{3}$, $\nu=4 \times 10^{13} \mathrm{~s}^{-1}$.

Graphical analysis of the nonlinear absorption and temporal amplification characteristics of a semiconductor plasma medium considers a tolerable small phase mismatch to compose an experimentally viable situation. Prior condition to achieve a significant gain is that the pump field should be well above the threshold amplitude and well below the damage threshold of the crystal [18]. Estimated threshold pump intensity for the onset of SPS is found to be $\approx 10^{14} \mathrm{~W} / \mathrm{m}^{2}$, which fulfils both these criteria.

Figure 1 displays the variation of nonlinear absorption coefficient $\alpha$ with respect to phase mismatch factor $\Delta k$ with scattering angle $\theta$ as a parameter. It is evident that there is gain at almost whole range of $\Delta k$. Identical variation has been observed for all the three different scattering angles. In fact tolerable $\Delta k$ leading to highest gain could be traced as nearly equal to $5 \times 10^{7} \mathrm{~m}^{-1}$. Hence

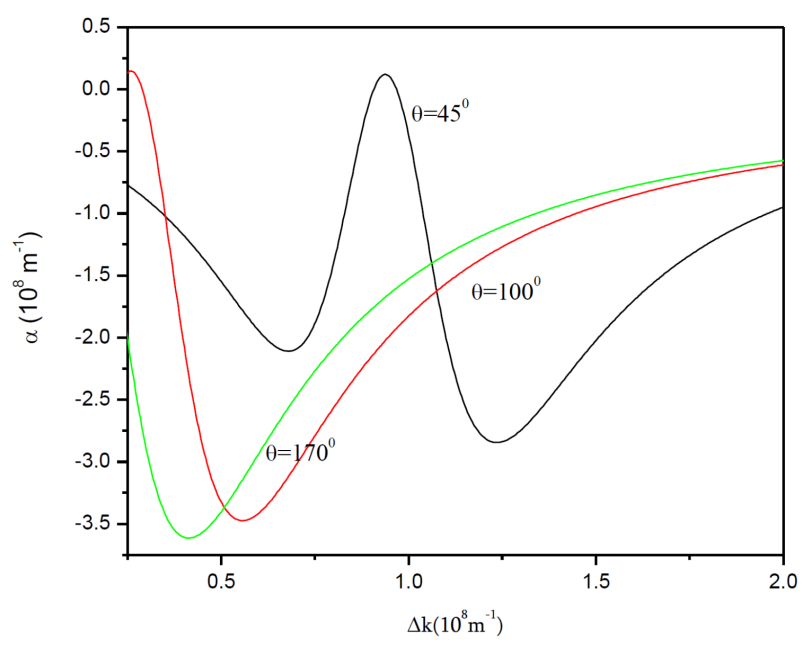

Fig. 1. Calculated phase mismatch dependence of nonlinear absorption coefficient with scattering angle $\theta$ as a parameter at $\omega_{p}=0.63 \omega_{0}$ and $E_{0}=4 \times 10^{8} \mathrm{~V} / \mathrm{m}$.

$\theta=170^{\circ}$ is found to be favourable for the consideration. Resonance between $k_{0}$ and $k_{1}$ leads towards minimum absorption coefficient. Initially when $k_{0}>k_{1}$, increasing scattering angle is found to shift highest gain position towards lower $\Delta k$ values. However, interaction length is considered to be higher than coherence length $l_{c}$ for the whole range of phase mismatch factor with $\Delta k<2 k_{0}$.

Figure 2 depicts the behaviour of transient gain coefficient $g_{T}$ as a function of plasma frequency $\omega_{p}$. These identical curves signify rapid increments in transient gain till $\omega_{p}=0.25 \omega_{0}$ and beyond this value further increments in $\omega_{p}$ cause almost stable transient derivatives. In the case of interaction length greater than the coherence length, higher transient gain is achieved for $L>l_{c}$, indicating energy flow from the pump to the idler wave for one coherence length, and strongest interaction with mutual transformation of pump wave and idler wave.

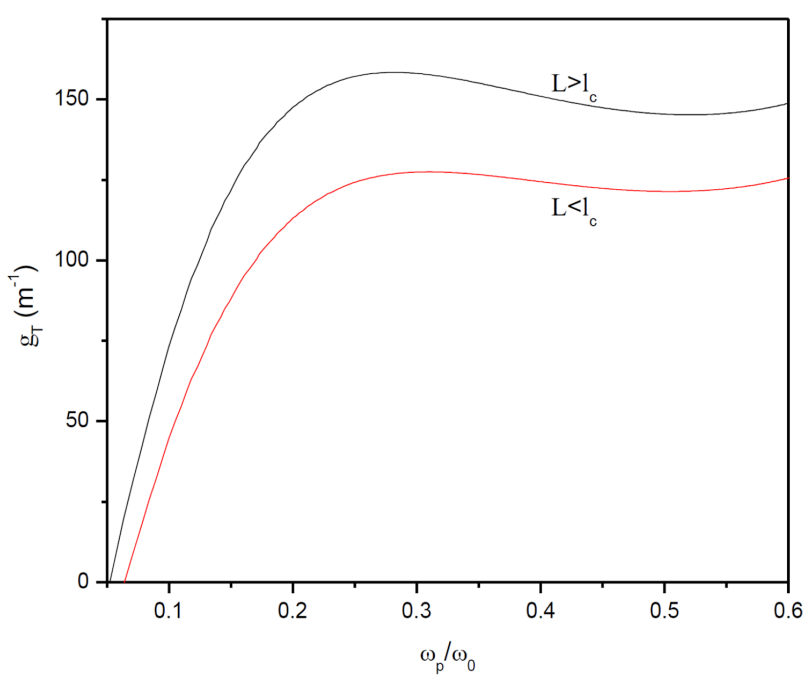

Fig. 2. Variation of the transient gain coefficient $g_{T}$ with plasma frequency at $E_{0}=4 \times 10^{8} \mathrm{~V} / \mathrm{m}$. 


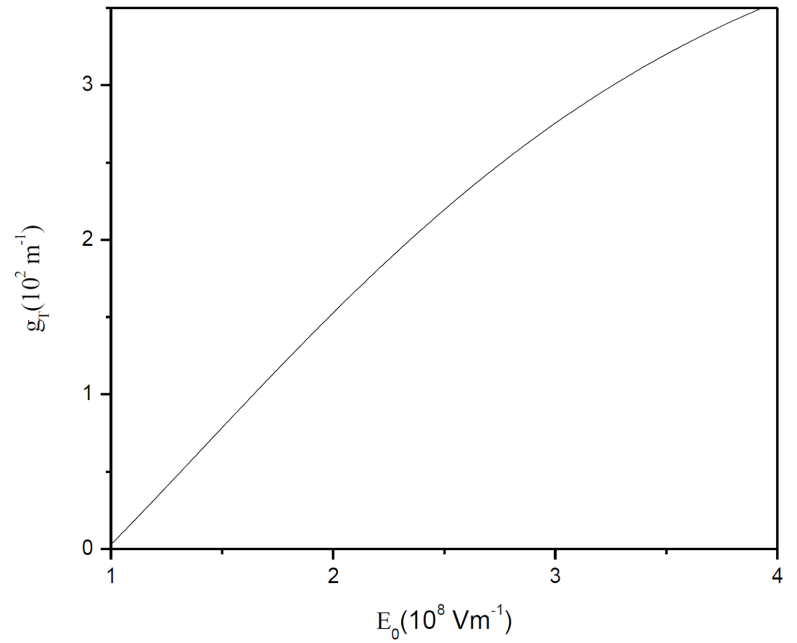

Fig. 3. Variation of the transient gain coefficient $g_{T}$ with pump amplitude $E_{0}$ at $\omega_{p}=0.63 \omega_{0}$.

In Fig. 3 transient gain coefficient $g_{T}$ is plotted against the pump amplitude $E_{0}$. Linear relationship between the pump amplitude and transient gain gets established through this curve.

Figure 4 depicts the qualitative behavior of the transient gain coefficient $g_{T}$ as a function of pulse duration at different interaction lengths. A close inspection of the figure reveals that the behavior of $g_{T}$ with increasing pulse durations is identical for all the interaction lengths. Firstly, $g_{T}$ increases with $\tau_{p}$ attaining maximum value which remains nearly constant for certain range signifying the existence of quasi saturation regimes. Beyond this regime, $g_{T}$ diminishes very rapidly to zero. It is interesting to note from Eq. (10) that $g_{T}$ is proportional to the square root of both the interaction length and interaction time. Temporal spreading of the gain is observed as the interaction length increases. Transient gain washes out at longer pulse durations upon increasing interaction length. Increments in interaction length shift the vanishing point of transient gain towards longer pump pulse durations. This behavior agrees well with the experimental observations [19].

Figure 5 depicts correlation between transient gain and steady state gain coefficient for different widths. For all widths $g_{T}$ is smaller than $g_{s s}$. The curves are identical and displaced towards higher transient gain as $\Gamma \tau_{p}$ is increased, due to the fact that in the transient case the gain is proportional to the total integrated energy [17]. This result shows the trend expected from the general temporal behavior and is in good agreement with Carman et al. [20].

Investigations of temporal amplification characteristics of semiconductor medium has been the primary aim of this study. Transient response of the order of a few microseconds is predicted theoretically. Favorable temporal amplification characteristics of the SPS in semiconductor plasma medium could be achieved with the specific control on relevant parameters such as pump amplitude, doping concentration, interaction length.

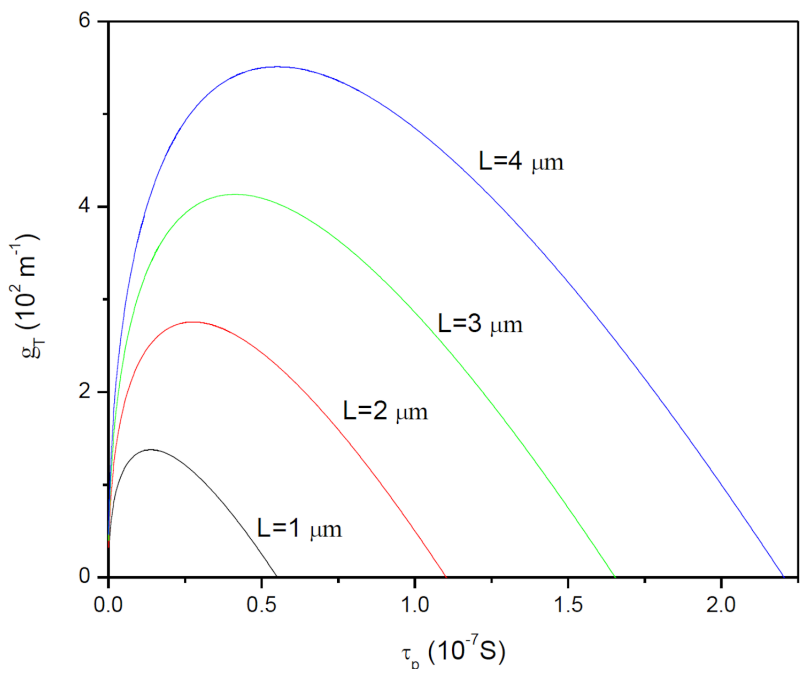

Fig. 4. Temporal variation of the transient gain coefficient with $L$ as a parameter at $\omega_{p}=0.63 \omega_{0}$ and $E_{0}=4 \times 10^{8} \mathrm{~V} / \mathrm{m}$.

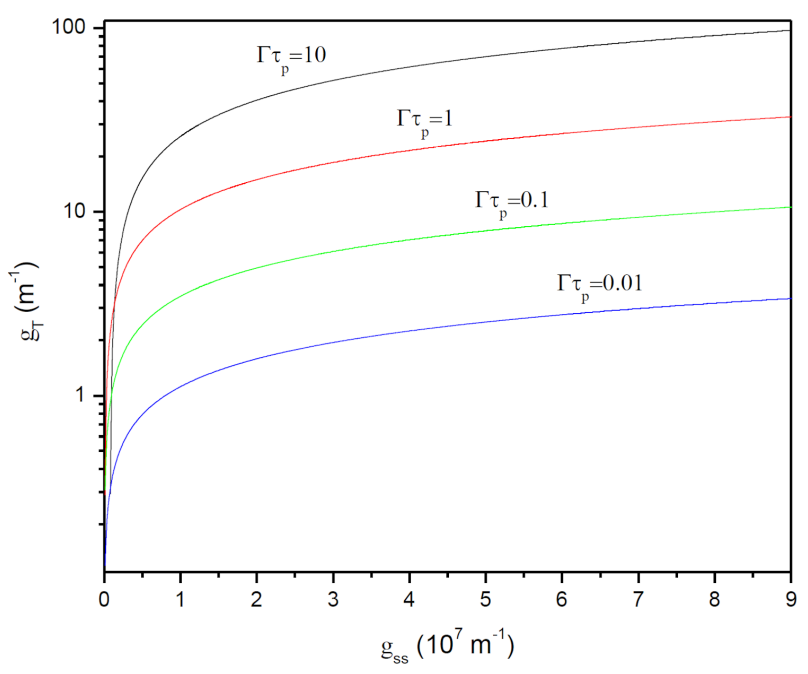

Fig. 5. Variation of the transient gain coefficient with steady state gain coefficient at $\omega_{p}=0.63 \omega_{0}$ and $E_{0}=$ $4 \times 10^{8} \mathrm{~V} / \mathrm{m}$.

Present analysis suggests the use of laser having pulse duration $\approx 10^{-8}-10^{-6} \mathrm{~s}$ which is readily available through $\mathrm{CO}_{2}$ laser. It is worth noting that interaction length of about a few $\mu \mathrm{m}$ is found to be favorable for maximum transient gain coefficient. Temporal spreading of the gain upon increasing interaction length could be used to tune the temporal behavior of medium.

Transient SPS is characterised by a wide spectrum and lower value of gain is obtained as compared to steady state scattering. Furthermore, we find good qualitative agreement with the experimental results.

\section{Conclusion}

The most remarkable feature of the reported results is the reduced magnitudes of required pump intensity at which stimulated parametric scattering gets initiated. 
The question of laser damage is central to the design and operation of nonlinear optical devices, due to the involvement of large radiation intensities shined over nonlinear crystals. Reduced pump requirements take care of the damage threshold of the crystal, too. Therefore, the present study successfully establishes an appropriate model helpful in the construction of a cost effective optical parametric amplifier with tunable steady state and temporal amplification characteristics.

\section{Acknowledgments}

One of the authors (Kamal Jain) acknowledges the financial assistance from the University Grant Commission, New Delhi, India, under Maulana Azad Minority Fellowship scheme.

\section{References}

[1] R.W. Boyd, Nonlinear Optics, Academic Press, New York 2003.

[2] Y.R. Shen, The Principles of Non-Linear Optics, Wiley, New York 1984.

[3] J.A. Armstrong, N. Bloembergen, J. Ducuing, P.S. Pershan, Phys. Rev. Lett. 127, 1918 (1962).

[4] A.V. Smith, Opt. Lett. 26, 719 (2001).

[5] M.M. Fejer, G.A. Magel, D.H. Jund, R.L. Byer, IEEE J. Quant. Electron. 28, 2631 (1992).

[6] M.B. Raybaut, R. Haidar,P. Kupecek, P. Lemasson, E. Rosencher, Nature 432, 374 (2004).
[7] A. Major, F. Yoshino, I.Nikolakakos, J.S. Aitchison, P.W.E. Smith, Opt. Lett. 29, 602 (2004).

[8] Zh. Kudyshev, I.Gabitov, A. Maimistov, Phys. Rev. A 87, 063840 (2013).

[9] A. Bahabad, M.M. Murnane, C.H. Kapteyn, Nature Photon. 4, 570 (2010).

[10] S.Y. Chen, A. Maksimchuk, E. Esarey, D. Umstadter, Phys. Rev. Lett. 84, 5528 (2000).

[11] I. Biaggio, V. Coda, G. Montemezzani, Phys. Rev. A 90, 043816 (2014)

[12] D.N. Klyshko, A.N. Penin, B.F. Polkovnikov, Sov. Phys. JETP 11, 11 (1970).

[13] B.V. Kryzhanovskii, Sov. J. Quant. Electron. 17, 7 (1987).

[14] A. Neogi, S. Ghosh, Phys. Status Solidi B 152, 691 (1989).

[15] S. Dubey, S. Ghosh, K. Jain, IOP Conf. Series J. Phys. Conf. Series 836, 012018 (2017).

[16] A. Yariv, Quantum Electronics, Wiley, New York 1975.

[17] C.S. Wang, in: Quantum Electronics, Eds. H. Rabin, C.L. Tang, Academic Press, New York 1975, part A.

[18] M.J. Soileau, E. William, W. Williams, E.V. Stryland, M.A. Woodall, Appl. Opt. 21, 4059 (1982).

[19] D.V. Linde, M. Maier, W. Kaiser, Phys. Rev. 178, 11 (1969).

[20] R.L. Carman, F. Shimizu, C.S. Wang, N. Bloembergen, Phys. Rev. A 2, 60 (1970) 